\title{
Effects of fenugreek seed powder on stress-induced hyperglycemia and clinical outcomes in critically ill patients: A randomized clinical trial
}

\author{
Akram Kooshki ${ }^{1}$, Zaher Khazaei ${ }^{2}$, Mojtaba Rad ${ }^{3}$, Azam Zarghi ${ }^{4}, *$, Akram Chanbari Mogaddam ${ }^{5}$
}

\begin{abstract}
${ }^{1}$ Associated Professor, Department of Nutrition and Biochemistry, Sabzevar University of Medical Sciences, Sabzevar, Iran

${ }^{2} \mathrm{MSc}$ of Epidemiology, Department of Health and Community Medicine, Faculty of Medicine, Dezful University of Medical Sciences, Dezful, Iran

${ }^{3} M S c$ of Faculty of Nursing erMidwifery, Sabzevar University of Medical Sciences, Sabzevar, Iran

${ }^{4} M S c$ of Faculty of Nursing \& Midwifery, Sabzevar University of Medical Sciences, Sabzevar, Iran

${ }^{5}$ Iranian Research Center on Healthy Aging, Sabzevar University of Medical Sciences, Sabzevar, Iran

Correspondence
\end{abstract}

Azam Zarghi, MSc of Faculty of Nursing \&Midwifery, Sabzevar University of Medical Sciences, Sabzevar, Iran

Email: a.zarghi59@gmail.com

History

- Received: 27 April 2018

- Accepted: 22 August 2018

- Published: 28 September 2018

DOI :

https://doi.org/10.15419/bmrat.v5i9.476

\section{Check for updates}

\section{Copyright}

(c) Biomedpress. This is an openaccess article distributed under the terms of the Creative Commons Attribution 4.0 International license.

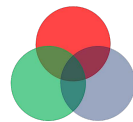

\section{ABSTRACT}

Introduction: Stress-induced hyperglycemia $(\mathrm{SIH})$ is commonly observed in critically ill patients and associated with poor clinical outcome for patients. Fenugreek seed powder has long been known as an anti-diabetic drug since its pharmaceutical properties were demonstrated. Materials: The study herein was a parallel, randomized controlled clinical trial consisting of 60 adult patients randomly divided into 2 groups ( $n=30$ per group). The study was conducted in Sabzevar, Iran in April 2015. The intervention group received $3 \mathrm{~g}$ of fenugreek seed powder by gavage, twice a day, in addition to routine care. The control group received only routine care. In the beginning, a daily evaluation of fasting and postprandial blood sugar was conducted for 10 days. Secondary components (prevalence of pneumonia; length of intensive care unit (ICU) stay, length of hospital stay, ventilator days, APACHE II score, and mortality rate) were measured until the time of hospital discharge or death. Data were analyzed via SPSS v.20 using Student's t-test (paired and unpaired), chi-square test, repeated measure ANOVA, and Wilcoxon test. Results: In during 10 days of treatment, there was a significant fall in mean glucose levels in 2 groups. However, this improve was more significant in Intervention group in compared to control group $(p<0.001)$. Conclusion: The present study suggests that daily diet with fenugreek seeds can be used as an add-on therapy with other medications in the management of SIH in critically ill patients.

Key words: Stress-induced hyperglycemia (SIH), Fenugreek seeds, Outcome, Intensive care unit (ICU)

\section{INTRODUCTION}

Stress-induced hyperglycemia (SIH) is a protective and transient clinical phenomenon, and a physiological adaptation. SIH is the result of increased sympathetic activity as well as increased release of antiinflammatory hormones and pro-inflammatory cytokines, thereby stimulating gluconeogenesis; it is thought to be a consistent body response ${ }^{1-4}$. SIH is common in intensive care unit (ICU) patients, is associated with poor prognosis and mortality, and is independent of the patient's diabetes history ${ }^{3}$. In stress-induced hyperglycemia, blood glucose level is randomly checked for $200 \mathrm{mg} / \mathrm{dL}$ or higher; hyperglycemia can also occur when the level of in-hospital fasting blood glucose is $126 \mathrm{mg} / \mathrm{dL}(7 \mathrm{mmol} / \mathrm{L})$ or higher $^{5}$. In these patients, factors that can impact the condition are total parenteral nutrition, dextrose supplementation, surgery, certain medications, insufficient insulin, and increased blood glucose levels ${ }^{6}$. In 2009, the American Association of Clinical Endocrinologists and American Diabetes Association (AACE/ADA), defined that the appropriate glucose level in critically ill patients should be maintained between 140 and $180 \mathrm{mg} / \mathrm{dL}(7.8 \text { and } 10.0 \mathrm{mmol} / \mathrm{L})^{7}$. SIH has undesirable effects on other body organs such as the cardiovascular system (acute myocardial infarction, cardiogenic shock and arrhythmia), Neurovascular (polyneuropathy) and immune system (immune system weakness, hospital stay, hospital infections), small and large bowel movements (gastroparesis, constipation or diarrhea), ischemic stroke and delay in wound healing ${ }^{4,8,9}$.

SIH may be a sign of impaired glucose tolerance, or insulin resistance and/or diabetes in the future ${ }^{10,11}$. Gastroparesis can occur in patients who are prone to hyperglycemia for a long time. It is an important issue in that most cases of gastroparesis are not curable and are usually a chronic condition ${ }^{12,13}$.

Hermanides and colleagues stated in a study that high glucose changes are hazardous, and that high glucose changes with high levels of glucose are associated with the highest mortality rates in the ICU ${ }^{14}$. In a study by Sung et al., patients with elevated blood sugar levels showed higher mortality rates than patients with normal blood sugar levels. In addition, it was found 
that SIH was associated with poor neurological outcomes and increased intracranial pressure in patients with brain trauma ${ }^{8}$. However, studies have shown that correction of hyperglycemia is specifically associated with a reduction in mortality, hospitalization time, mechanical ventilation and infection, and may improve clinical outcomes ${ }^{15}$. Despite improvements in blood glucose levels, there are still negative consequences, such as severe fluctuations in blood glucose, hypoglycemia, and potential increased risk for specific cancers; the latter remains the most important cause of morbidity and mortality.

While insulintherapy is currently used in the ICU to manage blood glucose, researchers have found that unlike chemical drugs and synthetic compounds, there have been few side effects reported with the use of herbal medicines. Thus, there is increasing interest in herbal agents. Researchers have found that agents that reduce insulin resistance might have better outcomes than agents that provide insulin ${ }^{16}$. It was seen that adjunct therapy gastrointestinal microbiome modulator (GIMM) increased the effectiveness of anti-hyperglycemic drugs and reduced the need for increased dosage of drugs. Furthermore, GIMM produces short-chain fatty acids instead of lactic acid in the body which reduces gastrointestinal complications of drugs ${ }^{17}$.

Indeed, fenugreek (herbal agent) has been and is a common herbal medicine in the field of diabetes ${ }^{18}$. Fenugreek (Trigonella foenum graceum) is a oneyear-old aromatic herb of the legume family. Fenugreek seeds (FSs) are known for having potent antioxidant properties as well as high nutritive, hypoglycemic, hypocholesterolemic, hyperinsulinemic and anti-diabetic activities. Traditionally, it was used as a galactagogue and to treat anorexia, fever gastritis, and gastric ulcers.

Renon et al. (in the early 20 century), Muller et al. (in 1924), René Gultier, Hoywer, and especially Mieno, prescribed fenugreek for revitalization in those who were extremely weak and thin due to food deprivation. It was also prescribed to prevent diabetic and pulmonary tuberculosis patients, who were prone to various diseases, from losing weight. They observed very good results. Thus far, many therapeutic effects of FSs have been observed in studies where most of the beneficial effects are attributed to the antioxidant, anti-inflammatory, and peri-biotic properties of the active components of the $\mathrm{FS}^{19}$.

The mechanism of the hypoglycemic effect of FSs is usually associated with the insulin signaling pathway. Fibers available in FSs, such as galactomannan and lignin, induce a decrease in blood glucose levels. Metabolite trigonelline nicotinic acid, saponin and amino acid 4-hydroxyisoleucine in FSs contribute to the anti-diabetic properties of the plant. Hydroxyisoleucine accelerates insulin secretion by pancreatic beta-cells and increases the effects of insulin on different tissues, such as muscle, fat, and liver. FSs inhibit alpha-amylase enzyme and inhibit the digestion and absorption of sugars, including starch in the gastrointestinal tract ${ }^{20}$.

It has been reported that the FSs are effective in lowering blood glucose levels in diabetic patients. Even after being cooked, its medicinal properties are still preserved. Kaur et al. (2016) examined the effect of FSs as an adjunct therapy with metformin in diabetic patients. In their study, Group 1 and group 2 were given $500 \mathrm{mg}$ of metformin twice a day while group 2 received $500 \mathrm{mg}$ metformin along with $1 \mathrm{~g}$ of fenugreek thrice a day. After 12 weeks, the results showed a significantly greater reduction in fasting and postprandial blood sugar levels in group 2 than in group $1^{18}$. Haeri et al. (2012) reported that FS, assisted by 4-hydroxyisoleucine, increases insulin secretion in hyperglycemic conditions and increases sensitivity to insulin and promotes anti-diabetic effects ${ }^{21}$. Furthermore, Losso et al. (2009) reported that eating baked goods, such as bread, made with fenugreek flour can help reduce insulin resistance in people with type 2 diabetes and ultimately improve carbohydrate metabolism. It has even been reported that the antidiabetic effect of FSs is comparable to the effect of the anti-diabetic drugs glibenclamide and metformin ${ }^{22}$, and is more effective than sitagliptin ${ }^{20}$.

This study evaluated management of glucose during hospital stays to promote better outcomes for the patients. In the present study, the aim was to evaluate the efficacy of FSs as an add-on therapy with insulin on SIH in ICU patients, and to compare the variations in mean glucose and clinical outcomes in those treated with a combination of FSs and insulin versus insulin alone.

\section{METHODS}

This parallel, randomized controlled clinical trial was conducted on adult patients admitted to two educational hospitals in Sabzevar, Iran from April 2015 to June 2016. The Institutional Review Board of Sabzevar University approved the study protocol (Code of ethics : IR.MEDSAB.REC.1394.128). Each patient by using random number table was assigned to control or intervention group and the same patient was placed in the opposite group. Each of the two groups (control and intervention) consisted of 30 patients. Conscious 
consent was obtained from all patients who had inclusion criteria, and all the risks and benefits of the study were explained. All patients received feeding by gavage with a standard solution prepared in the hospital. At the start of the study, a daily evaluation of fasting and postprandial blood sugar was done for 10 days. According to the consensus statement of the ACE and the ADA, hyperglycemia was confirmed in patients whose serum glucose levels rose higher than $180 \mathrm{mg} / \mathrm{dL}(10.0 \mathrm{mmol} / \mathrm{L})^{7}$.

The parameters that were measured were: incidence of pneumonia (and frequency), hospital length of stay, length of stay in the ICU, ventilator days, APACHE II score, mortality rate, and time until hospital discharge or death. Student's t-test (paired and unpaired), chisquare test, repeated measure ANOVA, and Wilcoxon test were used to determine the level of significance.

\section{Inclusion criteria}

Enteral nutrition order with Nasogastric Tube, age of at least 18 , serum glucose level $>180 \mathrm{mg} / \mathrm{dL}$.

\section{Exclusion criteria}

- Not satisfied to continue study

- History of hypersensitivity to fenugreek seed powder

- Pregnant females

- Bleeding disorders or INR (define INR) >2

- Patient with a history of surgery in the past 2 months

- Death before completing study

The primary outcomes included: Fasting Blood Sugar (FBS) and Postprandial Blood Sugar (PPBS).

The secondary outcomes included: Mortality rate, Ventilator days, Length of ICU stay, Length of hospital stay, APACHE II, and Frequent incidence of Pneumonia.

For enrollment of the 60 patients to the study, 116 patients were examined in terms of the early arrival and departure criteria of the study. During the research, out of 60 patients, 15 patients were excluded from the study on the basis of the departure criteria. Out of these 15 patients, 7 and 8 were placed in the control and intervention groups, respectively. Of the $7 \mathrm{ex}$ cluded patients in the control group, 3 patients were excluded due to death, and 4 patients were excluded due to dissatisfaction to continue the study. Out of the 8 excluded patients in the intervention group, 4 patients were excluded due to death and 4 patients were excluded due to dissatisfaction.

\section{Intervention}

The control group received only insulin injections via an infusion pump based on blood glucose levels and according to the insulin protocol ${ }^{23}$. The intervention group was given $3 \mathrm{~g}$ of FS powder mixed in warm soup twice a day, along with other medications, for 10 days. Patients were fed with similar nutrition formula every 3 hours for 24 hours. To achieve a doubleblinded study, the dishes of patients with gastric gavage were given a code. Patients received a syringe (60 cc) without a piston within 10-15 minutes at an altitude of 12 inches above the patient's stomach, and gavage was applied with intermittent manner and under the earth's gravity. After pouring warm soup into the patients' dishes, the researcher added $3 \mathrm{~g}$ of FS powder into the dishes of the intervention patients at 12 hours and 18 hours (without the presence of others). Addition of the FSs did not change the color or volume of the soup. The patients were investigated for FBS and PPBS every day for 10 days. The secondary outcomes were monitored until time of discharge or death of the patients.

The FSs used in this research were from the geographical area of Sabzevar, Iran. They were washed and dried in the vicinity of free airstream and then turned into powder.

\section{Statistical Analysis}

The data on the demographics of the clinical patients are presented in Table 2. Descriptive statistics using appropriate tests were used for all baseline characteristics. As determined by the data distribution, independent t-test or Mann-Whitney test was used to compare differences in the continuous variables between groups. Chi-square $\left(\chi^{2}\right)$ test was used for categorical variables. Statistical analysis was done using Student's paired and unpaired t-test. The paired $\mathrm{t}$ test was done to determine the significance within the groups and unpaired t-test was done to determine the significance between the groups. All P-values were two-sided, and significance was set at $\mathrm{p}<0.05$.

\section{RESULTS}

There were no significant differences between the 2 groups (intervention and control) in the first admission. The age range of the 60 samples ( 31 males and 29 females) was $25-65$ years. The mean age $(p=0.25)$, mean sex $(p=0.120)$, mean APACHE II score $(p=0.65)$ between the groups was not statistically significant. The mean baseline FBS level of the control group was $(162.10 \pm 11.17 \mathrm{mg} / \mathrm{dL})$ and for intervention group, it was $(164.13 \pm 17.80 \mathrm{mg} / \mathrm{dL})$ (Table 1). 
Table 1: Demographics and baseline variables of the intervention and control groups

\begin{tabular}{|c|c|c|c|c|}
\hline Variables & & Intervention & Control & P-Value \\
\hline \multirow[t]{2}{*}{ Gender } & Male & 12 & 19 & $0.12^{*}$ \\
\hline & Female & 18 & 11 & \\
\hline Age $($ Mean \pm SD $)$ & & $54.37 \pm 19.18$ & $59.53 \pm 17.37$ & $0.251^{* * *}$ \\
\hline \multirow{2}{*}{$\begin{array}{l}\text { Apache II score } \\
(\text { Mean } \pm S D)\end{array}$} & & $22.7 \pm 7.5$ & $23.7 \pm 8$ & $0.45^{\star *}$ \\
\hline & & & & \\
\hline \multirow{9}{*}{ The reasons for ICU stay } & Head trauma & 16 & 10 & $0.792^{*}$ \\
\hline & Multi-organ trauma & 9 & 7 & $0.771^{*}$ \\
\hline & Diabetes disease & 10 & 21 & $1.00^{\star}$ \\
\hline & COPD disease & 6 & 3 & $0.27^{*}$ \\
\hline & Heart disease & 19 & 17 & $0.792^{*}$ \\
\hline & Neurosurgical disease & 6 & 5 & $0.89^{*}$ \\
\hline & Digestive disease & 2 & 4 & $0.89^{*}$ \\
\hline & Glandular disease & 1 & 0 & $0.89^{*}$ \\
\hline & Infectious Disease & 3 & 2 & $0.89^{\star}$ \\
\hline \multicolumn{2}{|l|}{ Days spent in hospital } & $24.1 \pm 5.6$ & $27.4 \pm 6.6$ & $0.238^{* * *}$ \\
\hline \multicolumn{2}{|l|}{ Days spent in ICU } & $14.2 \pm 4.8$ & $17.6 \pm 6.7$ & $0.041^{* * *}$ \\
\hline \multicolumn{2}{|l|}{ Intestinal sounds } & $10.11 \pm 18.06$ & 20.20 & $0.414^{* *}$ \\
\hline \multicolumn{2}{|l|}{ Smoking } & 9 & 7 & $0.792^{*}$ \\
\hline \multicolumn{2}{|l|}{ Addiction } & 13 & 11 & $0.793^{*}$ \\
\hline \multicolumn{2}{|l|}{ Stimulant drugs gastric } & 13 & 11 & $0.793^{*}$ \\
\hline \multicolumn{2}{|l|}{ Sedative medications } & 15 & 14 & $1.00^{\star}$ \\
\hline \multicolumn{2}{|l|}{ GCS } & $7.6 \pm 2.06$ & $8.4 \pm 2.35$ & $0.275^{* * *}$ \\
\hline \multicolumn{2}{|l|}{ Volume of nutrition } & $154.66 \pm 53.54$ & $149.66 \pm 41.29$ & $0.724^{\star * *}$ \\
\hline \multicolumn{2}{|l|}{ Degrees ventilator } & $9.50 \pm 0.572$ & $9.76 \pm 0.63$ & $0.094^{* * *}$ \\
\hline \multicolumn{2}{|l|}{ Degree PEEP ventilator } & $0.932 \pm 6.40$ & $1.00 \pm 6.50$ & $0.694^{* * *}$ \\
\hline
\end{tabular}

PPBS levels were measured on the day of admission and then daily for 10 days. There was a significant reduction in mean PPBS levels of both groups. However, reduction in PPBS levels of the intervention group was greater than that of the control group $(\mathrm{p}<0.001)$ (Table 2).

FBS levels were measured on the day of admission then daily for 10 days. There was a significant reduction in mean FBS levels of both groups. However, the reduction in the mean FBS level in the control group was greater than that of the intervention group (intervention group: $164.17 \pm 1333.78 v s$. control group: $162.11 \pm 667.17 ; \mathrm{p}<0.001)$. An analysis of the mean percentage in FBS levels of both groups at the end of 10 days revealed a greater reduction in the intervention group $(\mathrm{p}<0.001)$ (Table 3$)$.

Secondary outcomes were evaluated too. Wilcoxon test revealed significant differences between the intervention and control groups with respect to prevalence of pneumonia [intervention group: 7 patients (23.3\%) vs. control group: 15 patients (50\%); $\mathrm{P}=0.03$ ] The average of days spent in ICU was significantly shorter in the intervention group than in the control group (intervention group: $14.2 \pm 4.7$ days $v s$. control group: $17.6 \pm 6.5$ days; $\mathrm{p}=0.028)$. The length of hospitalization was also significantly shorter in the 


\begin{tabular}{|c|c|c|}
\hline Time & Intervention group & Control group \\
\hline Day-0 & $222.12 \pm 73.44$ & $213.23 \pm 56.21$ \\
\hline First day & $219.11 \pm 86.7$ & $207.21 \pm 1.85$ \\
\hline Second day & $217.11 \pm 33.77$ & $204.17 \pm 66.84$ \\
\hline Third day & $214.12 \pm 33.06$ & $198.16 \pm 20.63$ \\
\hline Fourth day & $211.11 \pm 50.96$ & $194.15 \pm 7.4$ \\
\hline Fifth day & $209.12 \pm 33.12$ & $192.15 \pm 16.83$ \\
\hline sixth day & $205.11 \pm 73.91$ & $185.17 \pm 96.9$ \\
\hline Seventh day & $203.11 \pm 56.7$ & $183.12 \pm 1.26$ \\
\hline Eighth day & $200.11 \pm 266.7$ & $178.12 \pm 60.20$ \\
\hline Ninth day & $200.11 \pm 266.7$ & $178.12 \pm 60.20$ \\
\hline Tenth day & $195.11 \pm 32.96$ & $176.12 \pm 30.04$ \\
\hline
\end{tabular}

Table 3: Fasting blood sugar levels (Mean \pm SD) of the intervention and control groups throughout the study

\begin{tabular}{ccc}
\hline Time & Intervention group & Control group \\
Day-0 & $164.17 \pm 1333.78$ & $162.11 \pm 667.17$ \\
First day & $162.24 \pm 266.26$ & $158.11 \pm 56.12$ \\
Second day & $154.15 \pm 166.92$ & $156.10 \pm 54.78$ \\
Third day & $152.14 \pm 46.47$ & $154.10 \pm 55.64$ \\
Fourth day & $151.16 \pm 84.07$ & $152.10 \pm 20.67$ \\
Fifth day & $148.16 \pm 80.82$ & $150.10 \pm 61.24$ \\
Sixth day & $145.16 \pm 27.45$ & $148.10 \pm 61.20$ \\
Seventh day & $144.12 \pm 11.88$ & $146.10 \pm 4334.03$ \\
Eighth day & $141.10 \pm 24.12$ & $144.9 \pm 44.87$ \\
Ninth day & $137.11 \pm 37.01$ & $142.9 \pm 41.8$ \\
Tenth day & $133.9 \pm 84.31$ & $140.9 \pm 30.8$ \\
\hline
\end{tabular}

intervention group (intervention group: 24.1 \pm 5.6 vs. control group: $27.4 \pm 6.6 ; \mathrm{p}=0.041)$. The mean APACHE II score was not significantly affected by FSs. The ventilator days were not significantly different in the 2 groups ( $16.06 \pm 4.81$ for intervention group vs. $20.26 \pm 6.05$ for control group; $\mathrm{P}=0.64$ ). As well, mortality rates was not different $(2.2 \%$ vs. $20 \%$; $\mathrm{p}=0.12$ ). There were no adverse events attributable to the use of FSs in this study.

\section{DISCUSSION}

Stress-induced hyperglycemia is a metabolic disorder that is characterized by a high level of blood glu- cose. It is associated with undesirable and adverse clinical outcomes and is independently involved in the prognosis of the patient. In this study, we compared the anti-hyperglycemic effect of fenugreek seed powder as an adjunct to insulin, versus insulin alone, in the treatment of stress-induced hyperglycemia of ICU patients. We observed a significant improvement $(\mathrm{p}<0.05)$ in glycemic profile in both groups. However, the regulation of blood sugar was significantly evident in the intervention group as compared to control group $(\mathrm{p}<0.001)$.

Our findings are supported by studies, such as one conducted by Sharma et al., which surveyed the hy- 
poglycemic effect of FSs in 60 diabetes mellitus (DM) patients for 24 weeks. In their study, 25-g of FSs was given daily in two equal doses during lunch and dinner. The results showed that FSs acted as a moderator to bring the level of blood glucose closer to the normal level ${ }^{24}$. Lu et al. (2008) demonstrated similar results where they used FS powder as an add-on therapy alongside anti-hyperglycemic agents to treat Type $2 \mathrm{DM}$ patients for a 12 -week duration. At the end of their study, there was a statistically significant decrease in FBS, 2h PPBS, and glycated hemoglobin (HbA1c) in the group where FS powder was used as add-on therapy, compared to the control group (which received only the anti-hyperglycemic agent) $(\mathrm{p}<0.05)$. Moreover, similar findings were presented in the study conducted by Gupta et al. Their study surveyed the effect of $1 \mathrm{~g} /$ day of hydroalcoholic extract of fenugreek on the glycemic control and insulin resistance in Type $2 \mathrm{DM}$ patients for a period of 2 months. They showed a significant improvement in the glycemic profile of the DM patients ${ }^{25}$. In all of the above studies, the placebo was given to the control group. In our study, however, we uniquely coded the soup bowls blindly.

The highly nutritional, biochemical and pharmaceutical potential of fenugreek seed makes it a unique spice crop. Each $100 \mathrm{~g}$ fenugreek seed contains 4.63 $\mathrm{g}$ saponin (4.63 g/100 g). Indeed, saponin plays a key role in controlling blood glucose. It increases the content of fiber, amino acid hydroxyisoleucine, and major alkaloid trigonelline; it also activates enzymes and enhances insulin secretion, improves insulin sensitivity of different tissues, and reduces insulin resistance ${ }^{20}$. Other studies were conducted on patients hospitalized in the wards while our current study was conducted on patients admitted to the ICU. Indeed, stress levels are high among ICU patients and using high insulin dose can have prominent adverse effects. Our study showed that adjunct therapy of FS can increase the effectiveness of anti-hyperglycemic drugs and reduces the need for a high dose of insulin. Therefore, agents like FS are favorable to researchers as they have the ability to reduce insulin resistance ${ }^{16}$.

GIMM therapy produces short-chain fatty acids instead of lactic acid in the body which help reduce gastrointestinal complications that can arise from use of other drugs ${ }^{17}$. FS has been and is a common herbal medicine in the field of hyperglycemia regulation ${ }^{18}$. As mentioned, FSs are known to have antioxidant, nutritive, hypoglycemic, hypocholesterolemic, and antidiabetic properties, and have traditionally been used as a galactagogue and/or to treat other conditions, including anorexia, fever gastritis, and gastric ulcers.
However, in this study, we demonstrate an alternative use of FS as a complementary approach for hyperglycemic management. Thus, FS may have a beneficial role, especially in stress-induced hyperglycemia.

The main limitations of the study herein are the low sample size, the lack of measurement of HbAlc levels and glucose-enhancing hormones (such as glucagon and hydrocortisol), and the mental and psychological problems of the patients (which were not evaluated).

\section{CONCLUSIONS}

The present study showed that when fenugreek seed powder is used as add-on therapy with other medications, it has a significant effect on glycemic profile and clinical outcomes of hyperglycemic patients. Therefore, we conclude that fenugreek seeds may be a promising additional therapy for the management of blood glucose levels in ICU patients with stressinduced hyperglycemia. As fenugreek seeds are inexpensive and abundant, their use in conventional doses should not bear adverse side effects in the treatment of hyperglycemia (the only absolute contraindication is during pregnancy).

\section{COMPETING INTERESTS}

The authors declare no conflict of interest.

\section{AUTHORS' CONTRIBUTIONS}

MR, AZ, AK, ZKH and ACM; Formal analysis: AZ and MR; Funding acquisition: MR, AZ, AK, ZKH and ACM; Investigation: AZ, MR and AK Methodology: $\mathrm{MR}, \mathrm{AZ}$ and $\mathrm{AK}$; Project administration: AZ, MR and AK; Resources: MR, AZ, AK, ZKH, and ACM. Software: $\mathrm{ZKH}$ and AZ; Supervision: $\mathrm{AZ}, \mathrm{AK}$ and $\mathrm{MR}$; Validation: AZ, AK and ACM; Writing - original draft: AZ, MR and AK; Writing - review \& editing: MR, AK, AZ, ACM and ZKH.

\section{ACKNOWLEDGMENTS}

We are sincerely grateful to nursing research center and research vice-Dept. Sabzevar School of nursing and midwifery and also to the authorities and respected personnel of Sabzevar ICU departments who helped us with this study. We would especially like to thank dear patients and their families who contributed to this study. This article is based on a part of master's thesis on critical care nursing. Plan number: (26511).

\section{ABBREVIATIONS}

AACE: American Association of Clinical Endocrinologists

ADA: American Diabetes Association 
American Diabetes Association (AACE / ADA)

CI: Confidence Interval

FBS: Fasting Blood Sugar

FS: fenugreek seed

GIMM: gastrointestinal microbiome modulator

ICU: intensive care unit

PPBS : Post Prandial Blood Sugar

RCT: Randomized clinical trial

SD: Standard deviation

SIH: Stress-Induced Hyperglycemia

\section{REFERENCES}

1. Arshadi S, Bakhtiyari S, Haghani K, Valizadeh A. Effects of fenugreek seed extract and swimming endurance training on plasma glucose and cardiac antioxidant enzymes activity in streptozotocin-induced diabetic rats. Osong Public Health and Research Perspectives. 2015;6:87-93. Available from: DOI:10.1016/j.phrp.2014.12.007.

2. Knieriem M, Otto CM, Macintire D. Hyperglycemia in critically ill patients. The Compendium on Continuing Education for the Practicing Veterinarian. 2007;29:360-2.

3. Rau CS, Wu SC, Chen YC, Chien PC, Hsieh HY, Kuo PJ. StressInduced Hyperglycemia, but Not Diabetic Hyperglycemia, Is Associated with Higher Mortality in Patients with Isolated Moderate and Severe Traumatic Brain Injury: Analysis of a Propensity Score-Matched Population. International Journal of Environmental Research and Public Health. 2017;14:1340. Available from: DOI:10.3390/ijerph14111340.

4. Bosarge PL, Kerby JD. Stress-induced hyperglycemia: is it harmful following trauma? Advances in Surgery. 2013;47:287-97. Available from: DOI:10.1016/j.yasu.2013.03. 002.

5. Bosarge PL, Shoultz TH, Griffin RL, Kerby JD. Stress-induced hyperglycemia is associated with higher mortality in severe traumatic brain injury. The Journal of Trauma and Acute Care Surgery. 2015;79:289-94. Available from: Doi:10.1097/ ta.0000000000000716.

6. Hsu CW. Glycemic control in critically ill patients. World Journal of Critical Care Medicine. 2012;1:31-9. Available from: DOI:10.5492/wjccm.v1.i1.31.

7. Moghissi ES, Korytkowski MT, DiNardo M, Einhorn D, Hellman R, Hirsch IB, et al. American Association of Clinical Endocrinologists and American Diabetes Association consensus statement on inpatient glycemic control. Diabetes Care. 2009;32:1119-31. Available from: DOI:10.2337/dc09-9029.

8. Sung J, Bochicchio GV, Joshi M, Bochicchio K, Tracy K, Scalea $\mathrm{TM}$. Admission hyperglycemia is predictive of outcome in critically ill trauma patients. Journal of Trauma and Acute Care Surgery. 2005;59:80-83.

9. Saadat SMS, Bidabadi E, Saadat SNS, Mashouf M, Salamat F Yousefzadeh S. Association of persistent hyperglycemia with outcome of severe traumatic brain injury in pediatric population. Childś nervous system. 2012;28:1773-1777.

10. Bock G, Man CD, Campioni M, Chittilapilly E, Basu R, Toffolo G. Pathogenesis of pre-diabetes: mechanisms of fasting and postprandial hyperglycemia in people with impaired fasting glucose and/or impaired glucose tolerance. Diabetes. 2006;55:3536-49. Available from: DOI:10.2337/db06-0319.

11. Rao SS, Disraeli P, McGregor T. Impaired glucose tolerance and impaired fasting glucose. Am Fam Physician. 2004;69:1961-8.
12. Aderinto-Adike AO, Quigley EM. Gastrointestinal motility problems in critical care: a clinical perspective. Journal of digestive diseases. 2014;15:335-344.

13. Alipour Z, Khatib F, Tabib SM, Javadi H, Jafari E, Aghaghazvini L. Assessment of the Prevalence of Diabetic Gastroparesis and Validation of Gastric Emptying Scintigraphy for Diagnosis. Molecular Imaging and Radionuclide Therapy. 2017;26:17-23. Available from: DOI:10.4274/mirt.61587.

14. Hermanides J, Vriesendorp TM, Bosman RJ, Zandstra DF Hoekstra JB, Devries JH. Glucose variability is associated with intensive care unit mortality. Critical Care Medicine. 2010;38:838-42. Available from: DOI:10.1097/ CCM.0b013e3181cc4be9.

15. Yamada T, Shojima N, Noma H, Yamauchi T, Kadowaki T. Glycemic control, mortality, and hypoglycemia in critically ill patients: a systematic review and network meta-analysis of randomized controlled trials. Intensive Care Medicine. 2017:43:1-15. Available from: DOI:10.1007/s00134-016-45230 .

16. Elbagir MN, Eltom MA, Mahadi EO, Berne C. Pattern of longterm complications in Sudanese insulin-treated diabetic patients. Diabetes Research and Clinical Practice. 1995;30:59-67. Available from: Doi:10.1016/0168-8227(95)01146-3.

17. Rebello CJ, Burton J, Heiman M, Greenway FL. Gastrointestinal microbiome modulator improves glucose tolerance in overweight and obese subjects: A randomized controlled pilot trial. Journal of Diabetes and Its Complications. 2015;29:12726. Available from: DOI:10.1016/j.jdiacomp.2015.08.023.

18. Kaur M. IJBCP International Journal of Basic \& Clinical Pharmacology Research Article To study the efficacy and tolerability of fenugreek seed powder as add- on therapy with metformin in patients of type- 2 diabetes mellitus. International Journa of Basic and Clinical Pharmacology. 2016;5:378-83. Available from: DOI:10.18203/2319-2003.ijbcp20160748.

19. Ashish DP, Sancheti VP, Puja MS. Review article on fenugreek plant with its medicinal uses. SND Coll Pharmacy. 2014;4:20.

20. Koupý D, Kotolova H, Rudá JK. Effectiveness of phytotherapy in supportive treatment of type 2 diabetes mellitus II. Fenugreek (Trigonella foenum-graecum). Ceska a Slovenska farmacie: casopis Ceske farmaceuticke spolecnosti a Slovenske farmaceuticke spolecnosti. 2015;64:67-71.

21. Haeri MR, Limaki HK, White CJ, White KN. Non-insulin dependent anti-diabetic activity of $(2 \mathrm{~S}, 3 \mathrm{R}, 4 \mathrm{~S})$ 4-hydroxyisoleucine of fenugreek (Trigonella foenum graecum) in streptozotocininduced type I diabetic rats. Phytomedicine. 2012;19:571-4 Available from: DOI:10.1016/j.phymed.2012.01.004.

22. Lu FR, Shen L, Oin Y, Gao L, Li H, Dai Y. Clinical observation on trigonella foenum-graecum $L$. total saponins in combination with sulfonylureas in the treatment of type 2 diabetes mellitus. Chinese Journal of Integrative Medicine. 2008;14:56-60. Available from: DOI:10.1007/s11655-007-9005-3.

23. Agus MS, Wypij D, Hirshberg EL, Srinivasan V, Faustino EV, Luckett PM, et al. Tight Glycemic Control in Critically III Children. The New England Journal of Medicine. 2017;376:72941. Available from: DOI:10.1056/NEJMoa1612348.

24. Sharma RD, Sarkar A, Hazra DK, Misra B, Singh JB, Maheshwari BB. Hypolipidaemic effect of fenugreek seeds: A chronic study in non-insulin dependent diabetic patients. Phytotherapy Research. 1996;10:332-4. Available from: Doi:10.1002/ (sici) 1099-1573(199606)10:4<332::aid-ptr827>3.0.co;2-j.

25. Gupta A, Gupta R, Lal B. Effect of Trigonella foenum-graecum (fenugreek) seeds on glycaemic control and insulin resistance in type 2 diabetes mellitus: a double blind placebo controlled study. The Journal of the Association of Physicians of India. 2001;49:1057-61.

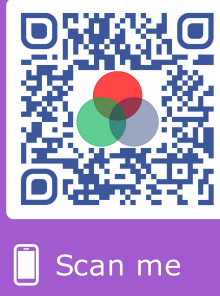

